University at Albany, State University of New York

Scholars Archive

7-2005

\title{
Reckoning the Shape of Everything: Underdetermination and Cosmotopology
}

\author{
P.D. Magnus \\ University at Albany, State University of New York, pmagnus@albany.edu
}

Follow this and additional works at: https://scholarsarchive.library.albany.edu/cas_philosophy_scholar

Part of the Philosophy of Science Commons

\section{Recommended Citation}

Magnus, P.D., "Reckoning the Shape of Everything: Underdetermination and Cosmotopology" (2005).

Philosophy Faculty Scholarship. 47.

https://scholarsarchive.library.albany.edu/cas_philosophy_scholar/47

This Article is brought to you for free and open access by the Philosophy at Scholars Archive. It has been accepted for inclusion in Philosophy Faculty Scholarship by an authorized administrator of Scholars Archive. For more information, please contact scholarsarchive@albany.edu. 


\title{
Forthcoming in the British Journal for the Philosophy of Science
}

\section{DRA I T}

Reckoning the Shape of Everything:

\section{Underdetermination and Cosmotopology*}

\author{
P.D. Magnus
}

June 18, 2004

\begin{abstract}
This paper offers a general characterization of underdetermination and gives a prima facie case for the underdetermination of the topology of the universe. A survey of several philosophical approaches to the problem fails to resolve the issue: The case involves the possibility of massive reduplication, but Strawson on massive reduplication provides no help here; it is not obvious that any of the rival theories
\end{abstract}

*The present paper is based on work presented at UC San Diego, Bowdoin College, and the University at Albany. I want to express my thanks to Thomas Baumgarte, Nancy Cartwright, Jonathan Cohen, Craig Callender, Sam Rickless, Ron McClamrock, Robert Howell, and an anonymous referee for helpful comments. 
are to be preferred on grounds of simplicity; and the usual talk of empirically equivalent theories misses the point entirely. If the choice is underdetermined, then the theories are not empirically equivalent! Yet the thought experiment is analogous to a live scientific possibility. Analysis of the cosmic microwave background might face underdetermination of this kind. So, it demands a reckoning. I suggest how the matter can be resolved, either by localizing the underdetermination or by defeating it entirely.

\section{Introduction}

Philosophers have fixed on the underlying geometry of space as a prime instance of the underdetermination of theory by evidence. Attention is usually directed to the metrical structure of space. As the argument goes, any non-Euclidean metric would be empirically equivalent to a Euclidean metric provided that there were appropriate universal forces to yield the observed 'inertial' trajectories. Quine gives the case as an instance of underdetermination, attributing it to Poincaré [Qui75, p. 322][BG90, p. 53]. Boyd offers it as "the example of experimentally indistinguishable, causally incompatible theories which has been paradigmatic at least since the publication of 
Reichenbach's Philosophy of Space and Time" [Boy73, p. 5]. Although Reichenbach also discusses the topological structure of space [Rei58, pp. 58-81], underdetermination of metrical structure has become the philosophical commonplace. This may be (in part) because discussions of the geometry of space in the 20th-century were prologomena to discussions of spacetime and relativity. Although relativity entails a non-Euclidean metric, it does not settle questions of topology.

Yet, in the bombastic language of a recent letter to the journal Nature, "Since antiquity, humans have wondered whether our Universe is finite or infinite. Now, after more than two millennia of speculation, observational data might finally settle this ancient question" $\left[\mathrm{LWR}^{+} 03\right.$, p. 595]. The threat of underdetermination arises: Can the data really settle the matter?

After characterizing underdetermination in general, I describe a thought experiment that gives the prima facie case for underdetermination here. A survey of some philosophical approaches to the problem shows that none of them resolve the issue. Since developments in recent cosmology connect the thought experiment with live scientific possibilities, however, it's as if the Swamp Man has knocked on our door. What to do? I argue that the matter can be resolved, either by localizing the underdetermination or by defeating 
it directly.

\section{A brief preliminary}

Underdetermination is sometimes taken to be the same as the problem of empirically equivalent rival theories, but let's think in broader terms. ${ }^{1}$ To put it crudely, we can say that underdetermination obtains when scientists are unable to responsibly decide which theory to believe. That is, the choice between rival theories is underdetermined if scientists cannot make a responsible choice of one over the others. Underdetermination is thus always relative to some standard for what will count as responsible theory choice. For specific cases of underdetermination, it is also helpful to distinguish the scope of the case - the range of circumstances across which responsible choice is impossible. In order to impress us, a case of underdetermination should obtain between rivals we take seriously, according to a standard that we find reasonable, and with a scope that includes not only our present circumstance but also most any circumstances we can expect to find ourselves in.

Thinking of underdetermination in this way allows us to represent it as a

\footnotetext{
${ }^{1}$ In $\S 4.3$, we'll see reasons not to think of the problem discussed here as a case of empirically equivalent rivals.
} 
three-place predicate: Choice among a set of rival theories $T$ is underdetermined by standard $R$ with respect to scope $S$. We can then represent specific underdetermination claims using this predicate and appropriate quantifiers. In the next section, I will offer a simple illustration where the rival theories are about the topology of space- in $\S 4.4$, we will see that analogous worries can be raised about the topology of spacetime. Prima facie, the case is one in which no possible observations could decide between the rival topologies. It seems as if the choice between the rival topological theories is underdetermined by reasonable scientific standards with a scope that includes all naturally possible circumstances.

Cases like this are sometimes offered as evidence for more widespread underdetermination; that is, for the claim that the choice among all or most rival theories is underdetermined in a similar way.

\section{Around the universe in 80 days}

Imagine you board a rocket ship and fly in a straight line away from Earth. After some time, you find yourself approaching Earth - or so it seems. It's a blue-green planet orbiting a yellow sun, matching the planet you left behind 
to any discernible degree of detail. You might think you've flown in a circle, but you check your instruments and conclude that indeed you've gone in a straight line away from Earth. Is this planet Earth? How could you tell?

Let $S_{1}$ be the theory that space is a finite cube wherein opposite sides are identified, such that anything reaching the top side would emerge on the bottom, anything reaching the back would emerge on the front, and anything passing to the right side would emerge on the left. ${ }^{2}$ If $S_{1}$ were true, then the planet would be Earth. Like the astronaut in figure 1, you'd have flown away from Earth and arrived back there.

Let $S_{2}$ be the theory that space is a finite volume with its contents repeated twice over. Space is connected as in $S_{1}$ but is larger, such that when you arrive at this blue-green planet you've made it half-way across the universe. If $S_{2}$ were true, you'd have arrived at the likeness of Earth and not at Earth itself. The situation would be like figure 2 .

What could you do to decide between $S_{1}$ and $S_{2}$ ? You might retrace your path to Earth and ask if you'd been seen coming the other way - if they saw you from Earth, then your journey had taken you to Earth and you could

\footnotetext{
${ }^{2}$ This is equivalent to supposing that space is a 3 -dimensional torus. You might worry instead that space is a Klein Bottle, that space is finite in one dimension and infinite in the others, or whatall else; such variants may be plugged into the discussion that follows mutatis mutandis.
} 

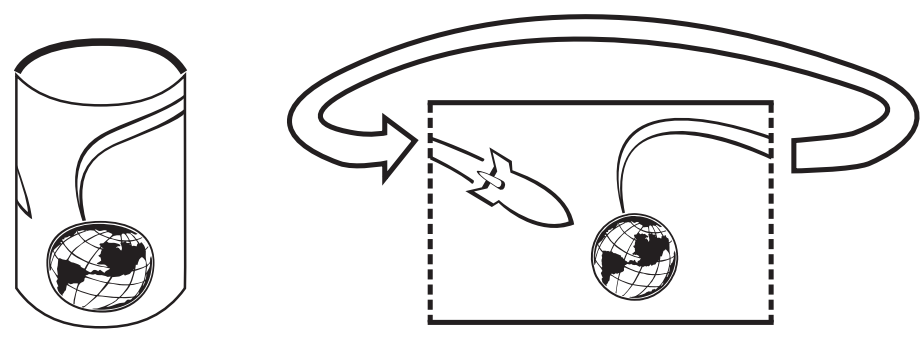

Figure 1: In finite space, the intrepid spaceman travels directly away from his planet only to arrive back home.

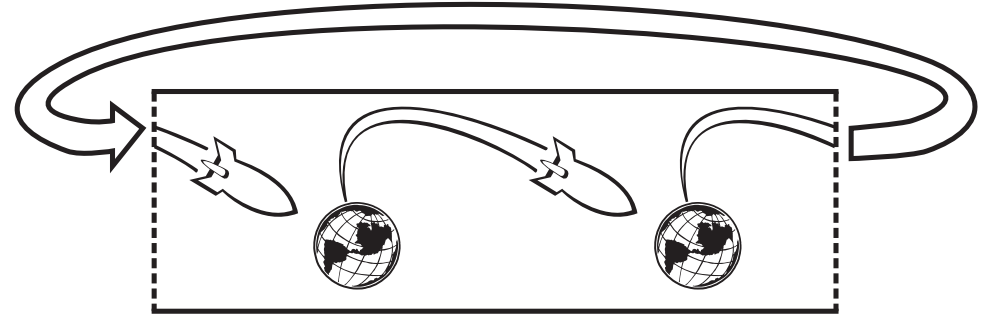

Figure 2: Space is larger but still finite. The intrepid spaceman travels directly away from his planet to arrive at an identical planet, while an astronaut leaving from the other planet travels to the first spaceman's home.

conclude that $S_{1}$ was correct. Yet how could they be sure it was you that they saw? If $S_{2}$ were true, the other planet would be an exact likeness, so it too would have sent out a rocket ship. Your friends on Earth would be unable to tell whether it was you or an indistinguishable likeness they had seen.

It looks as if your choice between $S_{1}$ and $S_{2}$ might be underdetermined for any evidence. Of course, you might entertain theories $S_{3}, S_{4}, S_{5}, \ldots$, in 


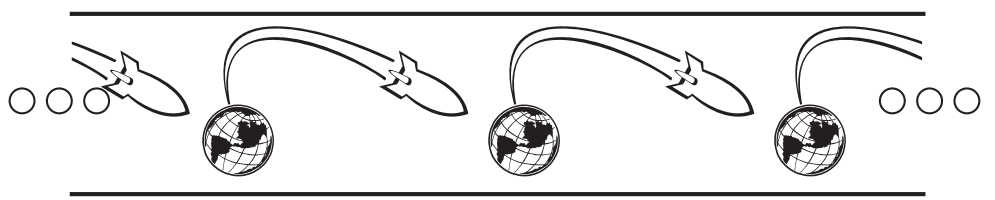

Figure 3: In infinite space, the intrepid spaceman travels to one of an infinite series of identical planets while an infinite number of other astronauts do the same.

each of which space is larger than in the last and there is one more planet sending out one more rocket. You may even entertain the limit case, $S_{\omega}$, in which space is infinite and there are an infinite number of indistinguishable planets launching an infinite number of rocket ships; see figure $2 .^{3}$

Let $\mathcal{S}$ stand for $\left\{S_{n}: 1 \leq n \leq \omega\right\}$. If your selection from $\left\{S_{1}, S_{2}\right\}$ is underdetermined, then your selection from $\mathcal{S}$ will similarly be underdetermined.

\section{Some attempts at resolving the scenario}

In this section, I consider four possible replies to the scenario and find them lacking.

\footnotetext{
${ }^{3}$ Transfinite theories of the form $S_{\omega+n}$ are ruled out; you can travel in either direction, so both the successor and the predecessor of each element must be defined.
} 


\subsection{Indexicality}

In Individuals, P.F. Strawson famously worries about the possibility of one sector of the universe repeating another down to the last detail. He calls this possibility "massive reduplication" [Str59, p. 20]. If our $S_{i}$ obtains for $i>1$, then massive reduplication would be realized. We should, then, consider whether Strawson's discussion sheds any light on the rivalry between the members of $\mathcal{S}$. He writes "that we build up our single picture of the world, of particular things and events, untroubled by possibilities of massive reduplications, content, sometimes, with the roughest locations of the situations and objects we speak of... This we do quite rationally, confident in a certain community of experiences and sources of instruction" [Str59, p. 289]. This seems to suggest that we might "quite rationally" accept $S_{1}$, but a moment's reflection will show that this is not so.

Strawson is initially worried about massive reduplication in the context of considering whether singular reference can be secured by means of descriptions. For any non-indexical description that we could know to hold of an object, he notes, we could not rule out the possibility that some other object also matches that description. Thus, massive reduplication arises as the worry that even a detailed description of a thing's environment might fail to 
individuate it if an indistinguishable thing-cum-environment exists elsewhere in the universe. Strawson resolves this worry by noting that we can employ indexical descriptions, picking out the thing as as 'this' or 'that', its environment as 'here' or 'there', and so on. He writes that to answer the worry about massive reduplication, "it is sufficient to show how the situation of non-demonstrative identification may be linked with the situation of demonstrative identification" [Str59, p. 20]. It is not always possible to indexically specify an object, for instance if it is far away and out of sight. Nevertheless, it is possible to say where the thing is and to specify that location relative to here. We can can pick out a thing by specifying its location in some sector of space, and the question of how that specification picks out one individual "may be answered by relating that sector uniquely to the sector which speaker and hearer themselves currently occupy" [Str59, p. 20]. Thus, for Strawson, we can always pick out an object with indexicals because we can place it in a unified spatiotemporal system.

It is important to notice that Strawson's argument does not show that massive reduplication does not obtain, nor does Strawson claim to show that - Strawson shows, at most, that the possibility of massive reduplication should not trouble our ordinary practices of identifying individuals. Your sit- 
uation after flying across the universe in your rocket ship is extraordinary, however, and may cause ordinary practice to break down. The morning before you leave on your journey, you know that you are in your house on your home planet, Earth - that Earth is the planet here and now. After your rocket journey, you arrive at a planet indistinguishable from your Earth. Imagine you land and go to a house indistinguishable from your house. Your key (which you brought with you) unlocks the door. You go inside. You climb into a bed like your bed in every detail and go to sleep.

The fact that you identify your home planet with an indexical - as your home planet - doesn't help you resolve whether this planet you arrive at is your home planet, whether this is your house, or whether this is your bed. In the scenario we are imagining, indexical reference to things back on the planet you left is unproblematic. Strawson thinks that having a single, spatio-temporal framework is required for referencing particulars. Since each of member of $\mathcal{S}$ posits a single spatio-temporal framework, Strawsonian considerations do not distinguish between them. The members of $\mathcal{S}$ disagree with one another as to what properties the framework would have, and that is the crux of the issue.

So it looks as if you have no way of knowing whether the bed you sleep 
in after you arrive is your bed at all. Of course, the residents of the planet on which you are sleeping are in no better position to decide between the members of $\mathcal{S}$ than you are. ${ }^{4}$ You have landed, gone into a house, and gone to sleep. If it is your house, then you have every right to do so. If it is not, then you are trespassing on the property of their heroic astronaut. Their heroic astronaut is on the next planet over sleeping in an identical bed, but what is that to them? If they believe $\neg S_{1}$, then they have grounds to arrest you.

If the problem is indeed underdetermined, then they will not have reasonable grounds to decide whether $S_{1}$ is true or not. They may adopt a fideist position and believe one of the members of $\mathcal{S}$ on faith, or they may adopt an agnostic position and refuse to affirm or deny any of the members of $\mathcal{S}$. If the former, they should welcome you if they are charmed by $S_{1}$ but arrest you otherwise. If the latter, their choice is not so easy. Although they don't wish to believe any member of $\mathcal{S}$, they are forced to act toward you in some way or other. They might reason in this way: Since no considerations could favor a member of $\mathcal{S}$ over any of the others, then they should assume that the members of $\mathcal{S}$ are equiprobable. ${ }^{5}$ They know that if $S_{1}$ is true, then you

\footnotetext{
${ }^{4}$ If $S_{1}$ is true, then the residents are we Earthlings. Otherwise, not.

${ }^{5}$ This appeal to the principle of indifference would be irresponsible of them, I suppose.
} 
are their hero, but if some other member of $\mathcal{S}$ is true, then you should be arrested. $S_{1}$ is measure zero in $\mathcal{S}$, so they may safely ignore that possibility. You are arrested for trespassing in the night, and you are forced to sell your rocket ship to pay legal fees. Tragic, no? ${ }^{6}$

\subsection{Simplicity itself}

It seems that in order to avoid arrest, you must show that the choice between members of $\mathcal{S}$ is not underdetermined and that $S_{1}$ is to be preferred. You note that if $S_{1}$ describes the universe as having $m$ objects in it, then $\mathrm{S}_{n}$ describes the universe as having $n \cdot m$ objects. Invoking Occam's Razor, you conclude that $S_{1}$ wins out. Yet the prosecutor may insist that Occam's Razor applies to kinds rather than to individuals and note that the ontological excess of $S_{\omega}$ consists of more things but no more kinds. He insists further that infinite space is sufficiently simpler than unbounded, finite space to justify believing that space is infinite whenever possible. Thus, he concludes, $S_{\omega}$ is to be preferred. Insofar as simplicity is an underanalyzed desideratum, it is unclear what the jury should make of these appeals.

\footnotetext{
${ }^{6}$ The tragedy is acute, since either you were jailed unfairly (if $S_{1}$ is true) or other poor astronauts are treated as roughly as you are (otherwise).
} 


\subsection{Empirical equivalence}

It may be tempting at this point to say that philosophers already have a category in which to file cases like this one: 'If members of $\mathcal{S}$ are adequate to the phenomena, then there is no way to decide between them. They are all empirically equivalent.' Unfortunately, this is simply untrue given any usual sense of empirical equivalence.

It is traditional to say that two theories are empirically equivalent if they entail all the same observation sentences. Evaluating the empirical equivalence of the members of $\mathcal{S}$ requires dividing observation sentences from other sentences. This has always been a contentious issue, but suppose that an observation sentence is one that describes observable objects; colloquially, it describes things you can get your hands on. ${ }^{7}$ Given $S_{1}$, you can truly say upon arriving to the planet, 'Here is Earth.' Given any other member of $\mathcal{S}$, you cannot make this observation. So with observation sentences characterized in this way, the theories would entail different observation sentences and so ipso facto would not be empirically equivalent.

\footnotetext{
${ }^{7}$ If observation sentences only reported introspective mental events, then the members of $\mathcal{S}$ would count as empirically equivalent - but then scientific theories would be on the same footing as Cartesian sceptical scenarios. Insofar as we are tempted to consider something as exotic as topology, we've overcome mundane worries about dreams and evil demons. For readers still tempted by the phenomenalist route, see [Psi99, pt. I].
} 
We might instead follow Quine [Qui75] and adopt a behaviorist conception of observation sentences. On Quine's account, two theories are identical if they share all the same empirical consequences and are intertranslatable. ${ }^{8}$ It is easy to see that $S_{1}$ and $S_{n}$ (for some $n$ ) will count as the same theory for Quine. To translate from $S_{1}$ to $S_{n}$, map the predicate ' $x$ is my Earth' onto ' $x$ is some earth,' and so on. To translate from $S_{n}$ to $S_{1}$, map the predicate ' $x$ is my Earth' onto ' $x$ is my Earth, and I have never travelled a great distance from it (or if I have I have circled back)'; map 'The astronaut is on his home planet' onto 'The astronaut can truthfully utter "This is my Earth" '; and so on. Thus (on Quine's account) the members of $\mathcal{S}$ are not distinct theories. But if these are no distinct rivals, there cannot be any choosing between them and a fortiori no underdetermination of that choice.

So if we try to assimilate this example as a case of empirical equivalence, something very strange happens: The members of $\mathcal{S}$ either count as empirically inequivalent or they count as all being the same theory. Neither outcome captures the underdetermination that seems to obtain between them.

One might think that the problem here is the sentential treatment of

\footnotetext{
${ }^{8}$ Regarding Quine's criteria of theory identity, see my [Mag03]. Quine later changed his mind on these matters, but the revisions need not concern us here - see his contribution to [BG90].
} 
empirical equivalence. Suppose we instead follow van Fraassen [van80], who considers theories to be sets of models or structures, and call theories empirically equivalent if they have the same observable sub-structures. Now, the planets in each of the members of $\mathcal{S}$ are observable, so each of the theories has different observational sub-structures. $S_{1}$ has a solitary planet Earth, $S_{2}$ has a pair of distinct planets 'Earth', and so on. Thus, the theories are not empirically equivalent. This consequence could be avoided by specifying the members of $\mathcal{S}$ in a language without an identity predicate, but then the theories will be satisfied by all the same models - they would be the same theory and not genuine rivals. Thus the same unhappy outcomes obtain if we understand empirical equivalence semantically rather than sententially.

The problem of reduplication does not fit well into the rubric of empirical equivalence: If the rivals come out as distinct, they count as empirically inequivalent. So, the choice simply cannot come out as underdeterminednot because you could decide between the members of $\mathcal{S}$, but because the rubric of empirical equivalence is not up to the task of describing the case. Were you to make this rocket journey, you would find such an analysis to be frivolous logic chopping. The sense of underdetermination developed here can make sense of the underdetermination in this case, providing a strong 
reason to favor it over the usual story about empirical equivalence.

\subsection{Is this just a philosopher's fantasy?}

One might respond to this example by noting that it is purely hypothetical. If you travelled away from Earth and found an Earth-like planet then you would be unable to decide between the members of $\mathcal{S}$. The antecedent is a fanciful narrative, so we should not get too excited about the consequent. An argument that relies on a complicated, counter-factual scenario shouldn't lead us to expect underdetermination all over. The case is uninteresting - one might say — not because the choice fails to be underdetermined, but because the underdetermination follows from features of the spectacular, fictional case.

This reply simply won't do. The example of your rocket journey is simpler than actual cosmology in several respects, of course, but similar difficulties may arise in the context of relativistic cosmology. You will never get in a rocket ship and travel across the universe, but spacetime might be multiply connected in detectable ways. The remainder of this section will discuss physicists' attempts to make these determinations.

At the end of the 19th century, Karl Schwarzschild suggested that we 
might look for distant images of our own galaxy [Sch00]. Suppose we looked out with our telescopes and saw images of the Milky Way repeated out into infinity - the astronomical equivalent of the astronautical scenario above. We might think either that a distant galaxy strongly resembles our galaxy or that, because of the geometry of space, a galaxy that appears to be in the distance is our galaxy. Schwarzschild explains:

One could imagine that as a result of enormously extended astronomical experience, the entire Universe consists of countless identical copies of our Milky Way, that the infinite space can be partitioned into cubes each containing an exactly identical copy of our Milky Way. Would we really cling on to the assumption of infinitely many identical repetitions of the same world? In order to see how absurd this is consider the implication that we ourselves as observing subjects would have to be present in infinitely many copies. We would be much happier with the view that these repetitions are illusory, that in reality space has peculiar connection properties so that if we leave any one cube through a side, then we immediately reenter it through the opposite side. [Sch00, p. 2544] 
He identifies an intuition that infinite repetition without identity is absurd. ${ }^{9}$ Yet this reassurance carries no logical force, and the absurdity of infinite repetition is not a manifest contradiction. $S_{\omega}$ is consistent and as much in agreement with the imagined evidence as $S_{1}$. Nothing Schwarzschild says disarms the prima facie underdetermination between $S_{1}$ and $S_{\omega}$. He speaks elsewhere in the essay of what is true or real, but here he speaks of our happiness with a certain view. This suggests fideism: Because the choice between $S_{1}$ and $S_{\omega}$ is underdetermined, we may believe whatever will make us happiest. Schwarzschild says nothing further to dispel the many worries one might have about this resolution to the problem. ${ }^{10}$

One might hope that the problem is a relic of the 19th century, swept away when classical space was replaced by relativistic spacetime. No such luck. ${ }^{11}$ Even in the relativistic context, we could follow Schwarzschild's suggestion and scan the heavens for multiple images of single objects. Admittedly, attempts to identify multiple images of the Milky Way face considerable obstacles. Because the images that travel further would take longer to arrive,

\footnotetext{
${ }^{9} \mathrm{He}$ thinks we would find finite space reassuring, since it would give us the prospect of having surveyed all of space just as we have surveyed all the Earth.

${ }^{10}$ As I argue in [Mag], fideism might disrupt the scientific community or lead scientists to develop poor habits of thought.

${ }^{11}$ Luminet, et al. provide an excellent informal introduction to these issues [LSW99].
} 
the images we could see now would portray the Milky Way at different times. Further, each successive image would be shifted and show the galaxy from a different angle. Attempts to reidentify quasars and galactic clusters have faced similar difficulties. Phenomena like gravitational lensing complicate matters further, because there would be multiple images of some objects even if space were simply connected. A recent review concludes that there is "little chance to recognize different images of a given object" [ULL00, p. 7].

Recent work has attempted to develop statistical tests to distinguish between observations of independent objects in simply connected space and repeated observations of the same objects in multiply connected space. The so-called crystallographic method analyzes catalogs of astronomical objects of a given type and plots the pairwise distances between them. For each multiply connected geometry, there is a characteristic distance between images of the same object. If the universe were a billion lightyears across, for instance, every object would repeat with a billion lightyears between repetitions. When the distances between all objects of that type were plotted on a histogram, the repetitions would create a spike in the graph at a billion lightyears.

Unfortunately, the crystallographic method relies on catalogs of astro- 
nomical objects. These catalogs are problematic in themselves, since the position of each object in real space must be inferred from angular position and redshift. Inferring from redshift to distance requires making cosmological assumptions [ULL00, p. 7]. Also, problems with gravitational lensing and the motion of objects remain, although one can hope that these effects are not so large as to wash out the repetition. ${ }^{12}$

Another promising method considers variations in the microwave background. Photons arriving from the limits of our observation have spent the same amount of time in transit and travelled the same distance, so their starting points form a sphere, called the last scattering surface. If space were multiply connected, then the last scattering surface would eventually cross itself [CSS98] [Wee98]. It would form circles where it overlapped itself. We would see each circle twice, once from each side. This is illustrated in figure 4. Analysis of this kind is especially promising, because it does not rely on problematic inferences from redshift to distance. Our observations of the cosmic background are still too imprecise to discern whether there is any overlapping, but it may only be a matter of time [Ino01] [Ell03] [LWR $\left.{ }^{+} 03\right]$.

There is no denying that work being done in this area is ingenious, but

\footnotetext{
${ }^{12}$ Hopefully, these effects would blunt rather than eliminate the spike in the histogram.
} 


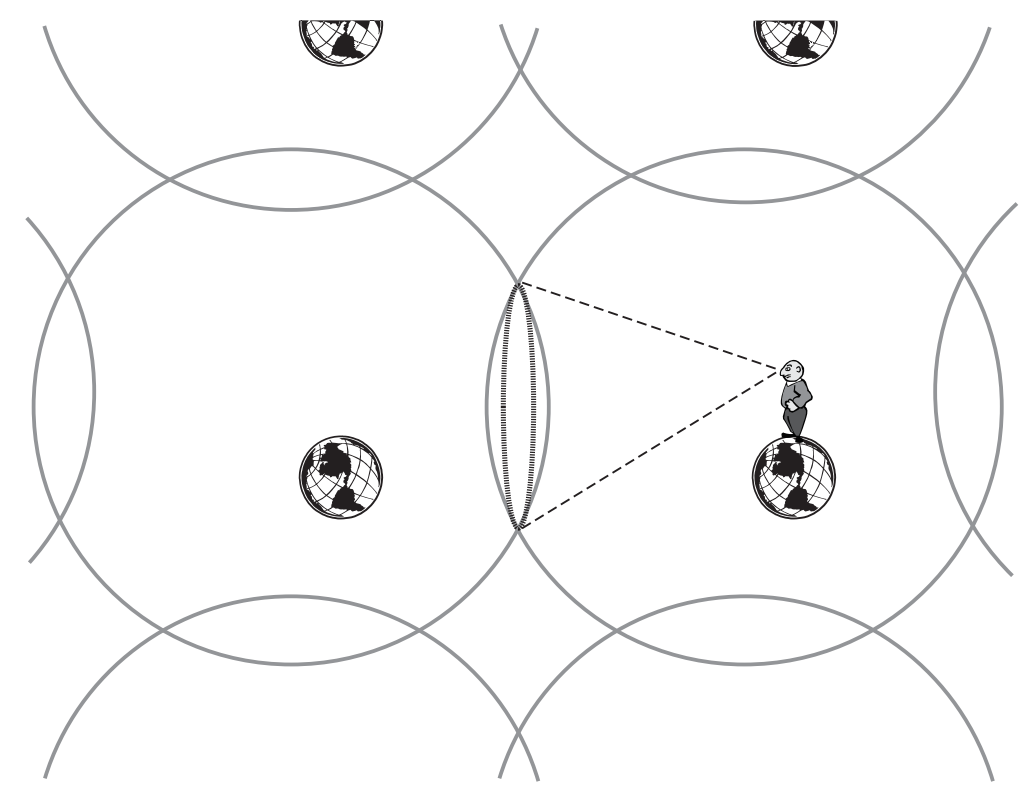

Figure 4: In multiply connected spacetime, the microwave background radiation would overlap with itself. The phenomenon would appear as rings in the background to an observer on Earth. 
it does nothing to resolve underdetermination. If the correlated pairs in our astronomical catalog exhibit certain features or if tell-tale patterns can be distinguished in the cosmic microwave background, physicists are prepared to conclude that the universe is multiply connected. In this, Schwarzschild correctly predicted what we would happily infer. Underdetermination scenarios like $S_{\omega}$ go unmentioned. Is this because contemporary physicists are fideists, as Schwarzschild seems to have been?

\section{Move along...}

One might think that the underdetermination reveals epistemological dry rot at the core of contemporary cosmology. That would surely be bad, but it wouldn't be a cause for general alarm if the example only showed something about cosmotopology. Given examples of underdetermination, though, some philosophers are quick to generalize and assume that the dry rot lies beneath the whole edifice of since. In this section, I will argue that such a generalization would be unjustified. In the next section, I will return to the example specifically and argue that worries are unjustified even there.

Suppose, though, that the example did exhibit a single instance of un- 
derdetermination. Perhaps we could look around and find a few other cases like it. Then, as John Earman writes, "the production of a few concrete examples is enough to generate the worry that only a lack of imagination on our part prevents us from seeing comparable examples of underdetermination all over the map" [Ear93, p. 31]. This worry plays on our suspicion that there is nothing special about the theory choices considered and found to be underdetermined, a suspicion that would incline us to think that this case is representative of underdetermination that hides everywhere.

Yet this same underdetermination cannot be all over. To see why, consider physical geometry stripped of any indexicals - call this non-demonstrative geometry. It would be a catalog of things and spatial relations: A planet of a certain local description is in such-and-so a configuration with respect to planets of identical local descriptions, and so on. (Following Strawson, I presume that removing the indexicals will eliminate any meaningful notion of numerical identity. If this is not the case, then it will be necessary to remove the identity predicate.) All members of $\mathcal{S}$ have the same non-demonstrative geometry. The possibility of massive reduplication is insufficient to make our choice of non-demonstrative geometry underdetermined. We may not be terribly interested in non-demonstrative geometry, but that's beside the point. 
It's enough to show that the underdetermination of physical geometry on account of possible reduplication doesn't show that all theory choice is underdetermined. Why should we suppose that other scientific theory choices are more like the choice of a physical geometry than like the choice of a non-demonstrative geometry?

Perhaps one still has an inchoate worry, but it becomes easy to sympathize with Kyle Stanford. Regarding each "hard-won particular alternative to an existing theory," he says "surely one or even a few such convincing cases do not provide sufficient warrant for concluding that genuine or serious empirical equivalence is a ubiquitous phenomenon!" [Sta01, p. S6]

\section{$6 \quad$...nothing to see here}

Note that a demonstrative geometry requires both a specification of the underlying geometry and a rule of repetition. Each member of $\mathcal{S}$ (except $S_{1}$ ) presumes a law-like connection between events on each of the Earths that preserves the reduplication: Each planet sends out an astronaut, each astronaut behaves in the same way, and so on. Since $S_{1}$ posits only one Earth, it does not require a rule of repetition. Note also that the underlying topology 
of space in $S_{1}$ and $S_{2}$ is the same; each is a torus. Since $S_{2}$ requires this topology and a rule of repetition, $S_{2}$ is just logically stronger than $S_{1}$. Thus, $S_{1}$ will always be better confirmed. ${ }^{13}$ Applying the same reasoning, $S_{1}$ is to be preferred over $S_{3}, S_{4}, \cdots$.

In this way, we can dispose of all the $S_{n}$ 's for $1<n<\omega$. Since $S_{\omega}$ has a different topology than $S_{1}$, it remains in contention. This justifies Schwarzschild's intuitions that $S_{1}$ and $S_{\omega}$ are the only real contenders. How can the physicists' implicit preference for $S_{1}$ be motivated? There has, historically, been a presumption of infinite, simply connected space (the topology of $S_{\omega}$ ). Since geometry has come to be an empirical matter, both simply connected and multiply connected space are contingent possibilities. The crucial difference isn't there.

$S_{\omega}$ posits an infinite repetition of the entities posited in $S_{1}$, and the difference between $S_{\omega}$ and $S_{1}$ amounts to the difference between believing or not believing in infinite repetition. That is the crux of the matter; if scientists have good reasons for eschewing claims of infinite repetition, then they have good reasons for preferring $S_{1}$.

\footnotetext{
${ }^{13}$ There may be reasons to prefer logically stronger theories in some cases (e.g., if they are predictively more accurate), but no such reasons are present here.
} 


\subsection{Rules of repetition}

$S_{\omega}$ 's requirement of infinite repetition amounts to a causal constraint that the infinitely many copies of each thing will behave in the same way. ${ }^{14}$ It's easy to see that a law of infinite repetition is either a sui generis kludge or a sceptical fantasy.

Relativity prohibits superluminal influences - that is, it's impossible to send a message at faster than the speed of light. ${ }^{15}$ Yet, given $S_{\omega}$, you could send a message instantaneously across space. Imagine you arrive at the next planet and want to send a message home. A radio message would take a very long time to cover that distance. So, instead, you write a message on a piece of paper. Now you drop the paper on the ground. The folks back home will not receive your sheet of paper- since it stays with you on the doppelganger Earth - but they will receive your message. Because of infinite repetition, another astronaut drops a similarly-marked sheet of paper on your planet Earth. You've sent the message instantaneously and without only a

\footnotetext{
${ }^{14}$ Reichenbach makes a similar point, remarking that "the topological properties of space are closely related to the problem of causality; we assume a topology of space that leads to normal causal laws" [Rei58, p. 80, emphasis in original].

${ }^{15} \mathrm{~A}$ different way of seeing the conflict: Relativity is usually taken to prohibit a general answer to the question of whether two space-like separated points are simultaneous; simultaneity is relative to reference frames. However, infinite repetition stipulates that what is happening here is happening in the same way just at this moment on all the other Earths. Thus, $S_{\omega}$ picks out a preferred reference frame, the frame in which repetition occurs.
} 
modicum of effort.

The situation is even worse than that. If $S_{\omega}$ were true, it would be possible to send these superluminal memos without ever leaving Earth. Supposing that sending a message involves an intention to communicate coupled with an appropriate action, you can send a message in this way: Form the intention to send a greeting to adjacent copies of yourself, write the 'Hello!' on the back of an envelope, and then read what you've written. The salutation, although inscribed by your hand, is actually a message from a far-away alien planet. The mind boggles.

This gives scientists good reason to reject $S_{\omega}$.

\subsection{Some possible replies}

One might try to defend $S_{\omega}$ by noting that quantum mechanics is also nonlocal. Since physicists accept quantum mechanics, why not infinite repetition? Although the relation between relativity and quantum mechanics is a complicated subject, ${ }^{16}$ the cases are very different. First, we have independent reasons for accepting quantum mechanics. It has been successful in many experimental domains. Second, experimental results preclude a local

\footnotetext{
${ }^{16}$ Maudlin [Mau94] provides a thorough discussion of these issues.
} 
alternative to quantum mechanics. $S_{\omega}$ has no independent motivation, and there is a local alternative (viz., $S_{1}$.) Third, although quantum mechanics picks out a preferred reference frame metaphysically, it does not do so epistemically. There is no way we could learn which reference frame is preferred. ${ }^{17}$ Determining the preferred reference frame in $S_{\omega}$ is trivial. Finally, quantum mechanics does not allow for super-luminal messaging. According to $S_{\omega}$, as we've seen, superluminal messaging should be child's play.

One might defend rules of repetition in a different way. In a deterministic universe, repetition need not be posited as a persistent causal law. Rather, it might obtain on account of special initial conditions: the contents of the universe were repeated $i$ times over at the beginning. Yet, a peculiar initial condition of this kind is as odd a duck as a law of infinite repetition. Perhaps it is even the same duck; given a regularity or best-system conception of laws, then special initial conditions of this kind just are laws. ${ }^{18}$ This is especially apparent when you consider that such an initial condition would designate a preferred reference frame, just as surely as a causal law of repetition would.

One might instead object that we have no way of knowing that the world

\footnotetext{
${ }^{17}$ Although this is not true of all interpretations of quantum mechanics, when true it is considered a virtue.

${ }^{18}$ This point is developed at greater length by Callender [Cal04].
} 
is not held together with perverse sui generis relations. Yet this just underscores the fact that underdetermination is relative to some standard. Given a standard that demands deductive certainty, the choice between the members of $\mathcal{S}$ will be underdetermined - but that standard would yield scepticism about most of science! Any standard of ampliative inference that warrants non-trivial conclusions will rely on background knowledge in some way. ${ }^{19}$ Given present background knowledge, this means that it will warrant rejecting laws of repetition.

\section{Conclusion}

The topological structure of space is an interesting case because the possible underdetermination can be presented in an intuitive way. I've argued that the matter really isn't underdetermined and (even if it were) there is no conclusion about all or most of science that follows from it. Along the way, I provided and deployed a characterization of underdetermination richer than those that define it in terms of empirical equivalence. The argument thus both resolves the underdetermination in this case and provides a reason to think of underdetermination using this richer characterization.

\footnotetext{
${ }^{19}$ Norton [Nor03] makes a general argument for this claim.
} 


\section{References}

[BG90] Robert Barrett and Roger Gibson, editors. Perspectives on Quine. Basil Blackwell, Cambridge, MA, 1990.

[Boy73] Richard N. Boyd. Realism, underdetermination, and a causal theory of evidence. Nô̂s, 7(1):1-12, March 1973.

[Cal04] Craig Callender. Measures, explanations and the past: Should 'special' initial conditions be explained? The British Journal for the Philosophy of Science, 55(2):195-217, June 2004.

[CSS98] Neil J. Cornish, David N. Spergel, and Glenn D. Starkman. Circles in the sky: finding topology with the microwave background radiation. Classical and Quantum Gravity, 15:2657-2670, 1998.

[Ear93] John Earman. Underdetermination, realism, and reason. In Midwest Studies in Philosophy, volume XVIII, pages 19-38. University of Notre Dame Press, 1993.

[EGS77] John Earman, Clark Glymour, and John Stachel, editors. Minnesota Studies in Philosophy of Science, volume VIII. University of Minnesota Press, Minneapolis, 1977. 
[Ell03] George F.R. Ellis. The shape of the universe. Nature, 425:566-7, October 92003.

[Ino01] Kaiki Taro Inoue. COBE constraints on a compact toroidal lowdensity universe. Classical and Quantum Gravity, 18:1967-1978, 2001.

[LSW99] Jean-Pierre Luminet, Glenn D. Starkman, and Jeffrey R. Weeks. Is space finite? Scientific American, pages 90-97, April 1999.

$\left[\mathrm{LWR}^{+}\right.$03] Jean-Pierre Luminet, Jeffrey R. Weeks, Alain Riazuelo, Roland Lehoucq, and Jean-Philippe Uzan. Dodecahedral space topology as an explanation for weak wide-angle temperature correlations in the cosmic microwave background. Nature, 425:593-595, October 92003.

[Mag] P.D. Magnus. Underdetermination, agnosticism, and related mistakes. Forthcoming in Inquiry.

[Mag03] P.D. Magnus. Underdetermination and the problem of identical rivals. Philosophy of Science, 70(5):1256-1264, December 2003. 
[Mau94] Tim Maudlin. Quantum Non-Locality and Relativity. Blackwell, Oxford UK, 1994. Aristotelean Society Series, v. 13.

[Nor03] John D. Norton. A material theory of induction. Philosophy of Science, 70(4):647-670, October 2003.

[Psi99] Stathis Psillos. Scientific Realism: How science tracks the truth. Routledge, London, 1999.

[Qui75] Willard Van Orman Quine. Empirically equivalent systems of the world. Erkenntnis, 9:313-328, 1975.

[Rei58] Hans Reichenbach. The Philosophy of Space $\mathcal{E}$ Time. Dover, New York, 1958. Translated by Maria Reichenbach and John Freund.

[Sch00] K[arl] Schwarzschild. On the permissible curvature of space. Vierteljahrschrift d. Astronom. Gesellschaft., 35:337-347, 1900. Translated by John M. Stewart and Mary E. Stewart. Printed in Classical and Quantum Gravity 15 (1998) 2539-2544.

[Sta01] P. Kyle Stanford. Refusing the devil's bargain: What kind of underdetermination should we take seriously? Philosophy of Science, 68 (Proceedings):S1-S12, September 2001. 
[Str59] P.F. Strawson. Individuals: An Essay in Descriptive Metaphysics. Routledge, London, 1959.

[ULL00] Jean-Philippe Uzan, Roland Lehoucq, and Jean-Pierre Luminet. New developments in the search for the topology of the universe, May 30 2000. http://arXiv.org/abs/gr-qc/0005128.

[van80] Bas C. van Fraassen. The Scientific Image. Clarendon Press, Oxford, 1980.

[Wee98] Jeffrey R. Weeks. Reconstructing the global topology of the universe from the cosmic microwave background. Classical and Quantum Gravity, 15:2599-2604, 1998. 